\title{
Die NIPT-Entscheidung des G-BA. Eine ethische Analyse
}

\author{
Christoph Rehmann-Sutter · Christina Schües
}

Eingegangen: 11. Februar 2020 / Angenommen: 15. Mai 2020 / Online publiziert: 14. Juli 2020 (C) Der/die Autor(en) 2020

Zusammenfassung Aus einer ethischen Perspektive analysieren wir die vom Gemeinsamen Bundesausschuss (G-BA) im September 2019 für Deutschland vorgelegte Änderung der Mutterschaftsrichtlinien, welche die Finanzierung der nichtinvasiven Pränataldiagnostik (NIPT) durch die gesetzlichen Krankenversicherungen unter bestimmten Bedingungen vorsieht. Die Regelung enthält vier wesentliche Elemente: eine Zielbestimmung (Vermeidung invasiver Testmaßnahmen), ein Zugangskriterium (der Test muss für die Schwangere ,geboten“ sein, um ihr eine Auseinandersetzung mit ihrer individuellen Situation zu ermöglichen), Aussagen zum Entscheidungsprozess (nach ärztlicher Beratung im Einzelfall) und eine in ihren Begründungen enthaltene normative Kontextualisierung (Schwangerschaftsabbruch nach $\$ 218$ a StGB).

Es zeigen sich Spannungen, die um zwei Achsen oszillieren: (1) Das befürchtete Leiden aufgrund der Geburt eines Kindes mit Trisomie oder dem Nichtwissen darüber kann letztlich nur subjektiv, aus der Perspektive der Schwangeren beurteilt werden. (2) Die Bedeutung der Einzelfallentscheidung bleibt unklar, weil für die Beurteilung von Einzelfällen auch allgemeine Gesichtspunkte maßgeblich sein müssen. Gerade in seiner Paradoxie und Flexibilität könnte, wie wir argumentieren, das Modell des G-BA aber eine gesellschaftspolitisch haltbare und ethisch letztlich vertretbare pragmatische Lösung darstellen.

Schlüsselwörter Nicht-invasive pränatale Tests (NIPT) · Pränataldiagnostik · Humangenetik · Gesetzliche Krankenversicherung · Gemeinsamer Bundesausschuss (GB-A)

Prof. Dr. phil., dipl. biol. C. Rehmann-Sutter $(\bowtie)$ · Prof. Dr. phil. C. Schües Institut für Medizingeschichte und Wissenschaftsforschung, Universität zu Lübeck, Königstraße 42, 23552 Lübeck, Deutschland E-Mail: rehmann@imgwf.uni-luebeck.de 


\title{
The decision of the German Federal Joint Committee to cover NIPT in mandatory health insurance. An ethical analysis
}

\begin{abstract}
Definition of the problem From an ethical point of view we analyse the ruling of the German Federal Joint Committee (Gemeinsamer Bundesausschuss, G-BA) of September 2019 to revise the guidelines about the coverage of noninvasive prenatal tests (NIPT) by mandatory health insurance, in order to include them under specified conditions.

Arguments The decision contains four essential elements: a definition of the aim of NIPT testing (to avoid invasive testing), a criterion of access (test must be "necessary" for the pregnant woman to tackle her individual situation), statements about the process of decision-making (after counselling by a physician and case by case) and a normative contextualization (termination of pregnancy under $\S 218$ a German penal law) which is to be found in its explanations.

Conclusion There are tensions in the proposed model that oscillate about two axes: (1) The suffering that results from a possible birth of a child with trisomy or from not knowing about the genetic condition of the fetus can ultimately only be evaluated subjectively, from the perspective of the pregnant woman. (2) The meaning of an individual decision in the case-by-case model remains unclear because when judging about individual cases general points of view also need to be considered. However, we argue that the model of the G-BA could be a sociopolitically and ultimately also ethically defensible pragmatic solution, exactly because of its paradoxes and its inherent flexibility.
\end{abstract}

Keywords Non-invasive prenatal tests (NIPT) - Prenatal diagnosis · Human genetics · Mandatory health insurance $\cdot$ Federal Joint Committee (Gemeinsamer Bundesausschuss, GB-A)

\section{Einleitung}

Am 19. September 2019 publizierte der Gemeinsame Bundesausschuss (G-BA) seine Entscheidung über Aufnahme der nicht-invasiven molekulargenetischen Pränataltests (NIPT) in den Leistungskatalog der Gesetzlichen Krankenversicherung (GKV). Die NIPT sollen demnach ,,in begründeten Einzelfällen und nach ärztlicher Beratung unter Verwendung einer Versicherteninformation“ eingesetzt werden können (G-BA 2019a). Ziel sei es, ,die zur Klärung der Frage des Vorliegens einer Trisomie 13, 18 oder 21 erforderlichen invasiven Untersuchungen - Chorionzottenbiopsie oder Amniozentese - und das damit verbundene Risiko einer Fehlgeburt nach Möglichkeit zu vermeiden" (G-BA 2019a).

Dieser Entscheidung ist eine rechtliche, politische und ethische Debatte vorausgegangen, sowohl um die Grundsatzfrage, ob in Deutschland die NIPT überhaupt in die reguläre Schwangerenversorgung mit Kostenübernahme durch die GKV aufgenommen werden sollen und unter welchen Bedingungen dies gerechtfertigt wäre, als auch darüber, ob NIPT nur innerhalb einer eingeschränkten Gruppe von Frauen 
übernommen werden sollen (Steger et al. 2018; Braun und Könninger 2018). NIPT sind in verschiedenen Formaten und von mehreren Firmen in Deutschland seit 2012 auf den Markt gebracht worden und seither nur als individuell zu bezahlende Leistung erhältlich (Schmitz 2013; Dohr und Bramkamp 2014). Das grundsächliche Argument für die Nutzung von NIPT ist, dass das Vorhandensein oder Nichtvorhandensein einer der häufigsten Trisomien $(13,18,21)$ und einer Reihe weiterer Befunde festgestellt werden kann, ohne durch einen Eingriff in den Uterus die Schwangerschaft einem Risiko einer Fehlgeburt auszusetzen. Auf der anderen Seite gab es Vorbehalte wegen selektiver Konsequenzen vor allem von Föten mit DownSyndrom.

Das Eingriffsrisiko der invasiven Diagnostik ist in den letzten Jahrzehnten kleiner geworden. Eine internationale Metaanalyse der publizierten Daten von 2000-2017 errechnete bei Amniozentese wie auch bei Chorionzottenbiopsie ein Abortrisiko von nur noch $0,35 \%$. Oft werden aber den Frauen höhere Zahlen mitgeteilt (Beta et al. 2018). Das Risiko variiert von Zentrum zu Zentrum und bleibt für die Frauen ein subjektiv entscheidungsrelevanter Faktor, der sie zur Durchführung eines risikofreien NIPT motivieren kann. Ein Problem beim direkten Vergleich der NIPT mit den invasiven Tests besteht aber darin, dass sich mit Letzteren ein größeres Spektrum von Chromosomenvariationen feststellen lässt als mit NIPT, welche vor allem auf die häufigsten Trisomien fokussieren. Außerdem gibt es weitere nicht-invasive pränataldiagnostische Verfahren wie das Ersttrimester-Screening und Ultraschalldiagnostik, bei denen wesentlich mehr Entwicklungsstörungen festgestellt werden können als mit NIPT. Insofern sollte man die verschiedenen Verfahren nicht nur hinsichtlich der Fehlerrate und des Abortrisikos vergleichen (BVNP 2020).

Die in Deutschland für die Entscheidung über die Aufnahme in den Leistungskatalog der Kassen zuständige Stelle, der G-BA, hatte im August 2016 zu NIPT für die Trisomien 13, 18 und 21 ein Methodenbewertungsverfahren in Gang gesetzt (G-BA 2016b), das von öffentlichen Kontroversen um die damit aufgeworfenen komplexen moralischen und gesellschaftspolitischen Fragen begleitet war. Im April 2019 hat eine Orientierungsdebatte im Bundestag stattgefunden. Eine Reihe von Bundestagsabgeordneten hat daraufhin den G-BA um eine Aussetzung der Beschlussfassung gebeten, um weiteren parlamentarischen Debatten nicht zuvorzukommen. Der G-BA ist dieser Bitte nicht gefolgt, hat jedoch den Bundestag aufgefordert, die Angelegenheiten zu erörtern und ggf. gesetzlich zu regeln (Brief von Josef Hecken vom 19.09.2019). Wie sich aus den Rahmenbedingungen der G-BA-Entscheidungen klar ergibt, konnte der G-BA keine nur ethische Entscheidung treffen, sondern musste den Nutzen der Methode bewerten (Huster 2017; Bornes 2020).

Umso wichtiger scheint es uns nun, diese rechtlich bindende Entscheidung des G-BA aus ethischer Sicht genauer zu untersuchen. Die im September 2019 festgelegte Zugangsregelung zur Kassenfinanzierung von NIPT ist bemerkenswert, weil sie eine eigentümliche Grundstruktur aufweist, die sich in einzelnen Punkten von den Regelungen in anderen Ländern unterscheidet: Es wird bewusst auf die Angabe einer numerischen Risikoschwelle als Zugangskriterium zu NIPT verzichtet und stattdessen auf die Klärung einer individuellen Belastungssituation der Schwangeren im Einzelfall gesetzt. NIPT soll zwar zur Vermeidung invasiver Diagnostik eingesetzt werden, aber dezidiert nicht Teil eines Routineablaufs bilden. 
Eine ethische Analyse dieser vom G-BA beschlossenen Regelung, die wir hier zur Diskussion stellen möchten, betrachtet ihre wesentlichen Elemente: Zielbestimmung, Zugangsregel und Aussagen zum Entscheidungsablauf. Außerdem wird teils implizit und teils explizit auf einen normativen Bezugsrahmen rekurriert, der hier berücksichtigt wird, insoweit er für die ethische Beurteilung wichtig ist. Die Regelung nimmt zwar auf die wichtigsten Anliegen der in der Debatte vertretenen moralischen Positionen Bezug, kann aber dennoch keiner dieser Positionen wirklich gerecht werden. Sie enthält Spannungen in zwei Hinsichten: (i) Zwischen der Absicht des G-BA, den Gebrauch von NIPT an möglichst klare Kriterien zu binden (die Abgrenzung zum Screening ,wird klar und eindeutig [...] geregelt“; G-BA 2019a) und einer nur subjektiv möglichen Bestimmung des psychosozialen Leidens und der entsprechenden Ungewissheit. (ii) Die Entscheidung im Einzelfall lässt weite Spielräume, die es einer Frau möglich machen, den Test dann zu benutzen, wenn sie aufgrund einer Trisomie 13, 18 oder 21 des zukünftigen Kindes eine für sie unzumutbare Situation befürchtet. Diese Regelung könnte aber - gerade in seiner Paradoxie und Flexibilität - eine gesellschaftspolitisch haltbare und letztlich auch ethisch für den deutschen Kontext wenig überraschende und vertretbare pragmatische Lösung darstellen. Die sie stützenden Gründe möchten wir in diesem Artikel aufzeigen.

\section{Sachverhalt}

Nicht-invasive Pränataltests (NIPT) arbeiten mit zellfreier foetaler DNA (cffDNA), die sich, wie Lo et al. (1997) nachgewiesen hatten, im Blutplasma einer schwangeren Frau finden lässt. Daraus können Informationen über das Erbgut des Fötus gewonnen werden. Die Tests wurden zunächst vor allem zur Entdeckung von fötaler Aneuploidie entwickelt (Chiu et al. 2008; Fan et al. 2008). Neben den häufigen autosomalen Trisomien 21, 18 und 13 können auch Fehlverteilungen der Geschlechtschromosomen bei Klinefelter, Turner, Triple X und XYY Syndrom (Hoyer 2018, S. 61) sowie die 22q11.2 Mikrodeletion (DiGeorge-Syndrom; IQWiG 2018, S. 2) festgestellt werden. Der Testumfang hat Erweiterungspotential, etwa für monogene Erbkrankheiten wie die Cystische Fibrose (Hill et al. 2014; Richter-Kuhlmann 2019). Die cffDNA wird mit Hochdurchsatztechnologien auf einer relativ niedrigen Auflösungsstufe sequenziert; es bietet sich deshalb prinzipiell die Möglichkeit, auch die komplette Genomsequenz des Fötus zu erstellen (zu den Trends vgl. Pös et al. 2019).

Der G-BA-Entscheid bezieht sich nur auf die drei Trisomien. Die Validität des Tests auf Trisomie 21 gilt als gut belegt, für Trisomie 13 und 18 gilt das mit Abstrichen (IQWiG 2018, S. iii). Deshalb eignen sich NIPT zumindest zu einer genaueren Wahrscheinlichkeitsbestimmung, um im Fall eines negativen Befundes für Trisomie 13, 18 und 21 auf einen invasiven Pränataltest (mit Amniozentese oder Chorionzottenbiopsie) verzichten zu können. Im Fall eines positiven Testergebnisses sollte, so die derzeitige Praxis, ein bestätigender invasiver Test (,diagnostischer Test") durchgeführt werden. Der Test kann bereits nach der vollendeten 9. Schwangerschaftswoche post menstr. durchgeführt werden. 
In der Schweiz ist 2015 die Kassenübernahme schon ab einem Ausgangsrisiko von 1:1000 beschlossen worden (vgl. Brauer et al. 2016), während NIPT in Großbritannien ab einem Ausgangsrisiko von 1:150 vom National Health Service kostenfrei angeboten wird (Sacco et al. 2019). In der öffentlichen Debatte, ob NIPT in Deutschland in die Regelversorgung aufgenommen werden soll, wurden nicht nur die Fragen der medizinischen Evidenz und der Wirtschaftlichkeit verhandelt, sondern es kamen auch ethische und gesellschaftspolitische Fragen ins Spiel: Ist NIPT ein Screening zur Vermeidung von Kindern mit Down-Syndrom? Oder dient der Test dazu, den Frauen ohne Zumutung eines Eingriffsrisikos die Voraussetzungen für eine selbstbestimmte und informierte Entscheidung über die Fortsetzung ihrer Schwangerschaft zu ermöglichen? Diese zwiespältige Wahrnehmung der Funktion der NIPT wurde von einer Reihe von parlamentarischen Voten in der Bundestagsdebatte vom 11. April 2019 bestätigt.

Bei der Entscheidung des G-BA vom 19.09.2019 (G-BA 2019b) handelt es sich zwar um die richtungsweisende Bestimmung, in der die Änderung der Mutterschaftsrichtlinie (Mu-RL; G-BA 2016a) festgelegt wurde, aber noch nicht um die Zulassung der Kassenfinanzierung. Die Kostenübernahme wird erst möglich, wenn der G-BA eine Versicherteninformation genehmigt hat, die seit Ende Februar 2020 als Entwurf vorliegt.

\section{Die Regelung des G-BA}

Der G-BA-Beschluss vom 19.09.2019 enthält vier Elemente, die gemeinsam seine normative Bedeutung ausmachen: Er enthält (i) eine Zielbestimmung der NIPT, (ii) ein Kriterium für das Angebot von NIPT in der Regelversorgung, (iii) eine Aussage zum Entscheidungsprozess und (iv) teils implizit, teils explizit (vor allem im Begründungsteil) eine normative Kontextualisierung. Die neue Regelung dient dazu, NIPT als sanftere Testmethode ohne Eingriffsrisiken im Rahmen der Regelleistungen in der GKV möglich zu machen - vor dem Hintergrund, dass die Amniozentese und Chorionzottenbiopsie bereits von der GKV übernommen werden. Sie will damit auch Finanzierungsgerechtigkeit herstellen. Andererseits will die Entscheidung, wie deutlich gesagt wird, vermeiden, dass der NIPT als routinisiertes Screening gegen chromosomale Variationen wie die Trisomie 21 eingesetzt wird.

\section{Zielbestimmung}

NIPT sollen ,„mit dem Ziel der Vermeidung“ der invasiven Tests durchgeführt werden. Diejenigen Frauen, bei denen NIPT keinen Befund ergibt, können dann auf die Durchführung eines invasiven Tests für dieselben Konditionen verzichten.

$\mathrm{Ob}$ und in wie vielen Fällen NIPT in der Praxis zum Verzicht auf invasive Tests führen, wird sich zeigen müssen. Eine Vermeidung der invasiven Tests durch vorangehende NIPT setzt ja voraus, dass der Testinhalt bei beiden Verfahren identisch ist. Dies ist aber nicht der Fall. NIPT testen wesentlich weniger Merkmale als invasive Tests (BVNP 2020). Die Vermeidung ist nur für diejenigen Frauen interessant, die 
bloß das wissen wollen, was ein NIPT abdeckt und wofür er hinreichend zuverlässig ist. $^{1}$

Mit dieser Bestimmung wird aber ein anderes Ziel ausgeschlossen, das mit NIPT rein technisch auch erreichbar wäre. Ravitsky (2017) nannte es das ,,public health rationale“: die Erhöhung der Detektionsrate von Schwangerschaften, die von einer testbaren Chromosomenvariation betroffen sind, oder die Verringerung der Anzahl von Geburten betroffener Kinder. Als Ziel der Finanzierungsregelung von NIPT wird nicht angegeben, die Zugangsschwelle zur genetischen Pränataldiagnostik (NIPT oder/und Amniozentese) herabzusetzen. Freilich wird es aber die finanzielle Hürde für diejenigen Frauen herabsetzen, die aus Gründen des Abortrisikos keine Amniozentese durchgeführt hätten, ohne zuerst eine NIPT zu machen.

\section{Zugangskriterium}

In den Mu-RL heißt es neu: „Bei besonderen Risiken oder zur Abklärung von Auffälligkeiten können im Einzelfall neben den üblichen Untersuchungen noch folgende Untersuchungen in Frage kommen. Dabei handelt es sich nicht um Screening-Untersuchungen.“ Mit „,folgende Untersuchungen“ sind dann u. a. die NIPT gemeint. Für diese seien die Vorgaben des Gendiagnostikgesetzes zusätzlich zu beachten. Dann stehen die folgenden zwei zentralen Sätze:

Der Test kann dann durchgeführt werden, wenn er geboten ist, um der Schwangeren eine Auseinandersetzung mit ihrer individuellen Situation hinsichtlich des Vorliegens einer Trisomie im Rahmen der ärztlichen Begleitung zu ermöglichen. Ein statistisch erhöhtes Risiko für eine Trisomie allein reicht für die Anwendung dieses Tests nicht aus. (G-BA 2019b, Nr. 3e)

Als weitere Voraussetzungen werden an anderer Stelle genannt: Das „Vorliegen des sonografisch bestimmten Gestationsalters und die Kenntnis der Anzahl der Embryonen oder Feten“. Der zweite oben zitierte Satz schließt aus, dass eine Risikoschwelle alleiniger Grund für die Durchführung eines NIPT ist; die in der humangenetischen Beratung übliche Risikobestimmung (aus Alter und Voruntersuchungen) muss aber gleichwohl einen Teil der für die Einschätzung relevanten Kriterien bilden, die im Einzelfall „ein besonderes Risiko“ ergeben.

Der erste zitierte Satz besagt, dass der Test ,geboten“ sein müsse, um der Schwangeren eine Situationsbewertung hinsichtlich Trisomien zu ermöglichen. Die Richtlinie spricht von der „Auseinandersetzung mit ihrer individuellen Situation“, zu welcher der Test ,geboten“ sein müsse. Was heißt in diesem Zusammenhang aber „geboten“? Der Test ist notwendig im Sinne einer epistemischen Dringlichkeit, um diese Auseinandersetzung zu ermöglichen: Die Frau braucht den aus dem Test zu erlangenden Befund notwendig, um ihre Situation einschätzen zu können. Das ist

\footnotetext{
${ }^{1}$ Im Rahmen dieses Artikels, der sich auf die Finanzierungsregelung konzentriert, verzichten wir auf eine Diskussion der klinisch-ethisch relevanten Fragen, in welchem Verhältnis NIPT zu anderen nicht-invasiven Methoden (Fein-Ultraschall) und Tests im Rahmen invasiver Verfahren (z. B. Microarray-Analyse) stehen soll. Vgl. dazu beispielsweise das Statement des BVNP (2020).
} 
dann der Fall, wenn sie in ihrer Lebenssituation einen schwerwiegenden Grund hat, wissen zu wollen, ob der Fötus eine Trisomie aufweist.

Es scheint uns schwer vorstellbar, dass mit dem Wort „geboten“ mehr gemeint sein sollte als diese epistemische Dringlichkeit. „Geboten“ soll gerade nicht im Sinn einer Pflicht verstanden werden, die als soziale oder moralische Norm verlangt, in bestimmten Situationen zu testen. Denn sonst wäre der Test ein Screening. Eine gesetzliche Norm zum Testen gibt es in Deutschland nicht. Im Gendiagnostikgesetz (§ 9) ist vielmehr das Recht auf Nichtwissen verbrieft.

Der Grund für eine Testung und für den Wunsch, Wissen über das Vorliegen von Trisomien zu erlangen, kann grundsätzlich in drei verschiedenen Situationen entstehen: (i) Wenn eine schwangere Frau ein Kind auch dann bekommen möchte, wenn es eine Trisomie hat, dies aber vorher wissen will, um sich darauf vorbereiten zu können. Oder (ii) wenn sie die Ungewissheit nicht erträgt - im Sinne der auch bei der invasiven Diagnostik für die Kassenfinanzierung anerkannten Angstindikation: starke Nervosität, Ängste, Schlafmangel, Partnerschaftskonflikt usw. -, ohne einen Abbruch in Erwägung zu ziehen. Der Grund kann weiterhin (iii) der sein, einen Schwangerschaftsabbruch im Fall des Vorliegens eines Befundes in Erwägung zu ziehen, weil in der Vorstellung die Geburt eines Kindes mit Trisomie für die Frau, die Familie und/oder für das Kind selbst zu einer zu schweren Belastung führen würde.

Auf die Situation iii (und nur darauf) richten sich die Erläuterungen in dem Dokument „Tragende Gründe“ (G-BA 2019c). In dieser Ausgangslage richtet sich die medizinische Notwendigkeit einer Diagnostik von Trisomien auf eine Situation der Schwangeren, in der sie die Frage klären muss, „ob durch die Schwangerschaft und deren Folgen eine [für sie] schwerwiegende Beeinträchtigung des seelischen Gesundheitszustandes oder eine Belastung erwächst“, die ,so schwer und außergewöhnlich sein [können], dass sie die zumutbare Opfergrenze übersteigen“ (G-BA 2019c, S. 4). Damit wird eine Parallele zur Regelung des Schwangerschaftsabbruchs hergestellt (ohne den Paragraphen aber zu nennen). Das ist eine dramatisch klingende Formulierung, deren Sinn sich nur aus dem normativen Kontext von $§ 218$ StGB erschließt (s. unten). - Im Lichte dieser Gesichtspunkte müssten die oben genannten ersten beiden Fälle eigentlich herausfallen, obwohl Situationen vom Typ ii auch zur Gesundheit der Schwangeren in Beziehung gesetzt werden können. Von der Formulierung in den Mu-RL (G-BA 2019b, Nr. 3e) wird ii nicht ausgeschlossen. Auch das Leiden am Nichtwissen kann ,eine Auseinandersetzung mit ihrer individuellen Situation hinsichtlich des Vorliegens einer Trisomie" notwendig machen, ohne dass damit das Leiden nach der Geburt eines Kindes mit Trisomie gemeint sein muss.

Ein weiterer Grund für diese erweiterte Auslegung der Regelung, dass sie auch Situationen vom Typus ii einschließt, besteht in Folgendem: Es kann nicht verlangt werden, dass eine nachfolgende invasive Testung und eventuell ein Schwangerschaftsabbruch bereits erwogen werden müssen, um sich für die Finanzierung von NIPT zu qualifizieren. Dies widerspräche dem Grundsatz, dass eine Entscheidung für jeweils den nächsten Schritt frei getroffen werden muss, ohne durch vorherige Festlegungen schon gebunden zu sein. Man kann deshalb der Frau vor einer NIPT keine Erklärung abverlangen, die besagt, dass sie im Falle eines Befundes auch den Abbruch der Schwangerschaft in Erwägung zieht. Somit muss das Leiden an Unge- 
wissheit als hinreichender Grund angesehen werden, um einen NIPT zu finanzieren. Dies müsste selbst dann der Fall sein, wenn die Frau versichert, keinen Schwangerschaftsabbruch in Erwägung zu ziehen. Das bloße Wissen-Wollen (Situation Typ i) dürfte hingegen kaum als hinreichender Grund angesehen werden, um NIPT durchzuführen, weil das Wissen-Wollen der NIPT-Ergebnisse allein nicht ausreicht, um zur Auseinandersetzung mit ihrer Situation ,geboten“ zu sein.

Es ist bemerkenswert, dass mit den neuen Mu-RL auch die Zugangskriterien für invasive Diagnostik auf die individuelle Situationseinschätzung und den Einzelfall umgestellt zu werden scheinen. Dies drängt sich vom Gesichtspunkt der Regelungslogik auf, weil nun zeitlich vor einer invasiven Diagnostik immer die NIPT möglich sein sollen. Allerdings wird u.E. nicht ausgeschlossen, dass eine Amniozentese oder Chorionzottenbiopsie auch ohne vorangehende NIPT durchgeführt werden können. Der erste zitierte Satz ist eine Kann-, keine Muss-Formulierung: „Test kann dann durchgeführt werden, wenn ...“ Der Grund dafür ist u.a., dass nicht nur NIPT, sondern auch andere nicht-invasive Verfahren (Fein-Ultraschall) zu einem hinreichend hohen Befundrisiko führen können, um eine invasive Testung nahezulegen.

Eine bestätigende invasive Testung nach einem positiven Befund aus NIPT wird von der Regelung nicht vorgeschrieben. Aber es muss zwingend darüber informiert werden, dass zur Bestätigung eines NIPT-Befundes vor der Entscheidung über einen Schwangerschaftsabbruch ein diagnostischer Test erforderlich ist. Ob die Frau diesen bestätigenden Test aber durchführen lässt, bleibt ihr überlassen.

\section{Entscheidungsprozess}

Der Einsatz von NIPT soll nach einer ärztlichen Beratung erfolgen, auf der Basis einer Versicherteninformation. (Diese liegt heute im Entwurf vor, soll mit Nutzerinnen getestet werden und wird für Ende 2020 in Aussicht gestellt.) Diese soll eine hinreichende, ausgewogene und intensive Aufklärung der Beratenen sicherstellen. Die Entscheidung über die (kassenpflichtige) Durchführung eines NIPT soll nicht nach allgemeinen Kriterien erfolgen, die wie eine Checkliste an die Schwangerschaften angelegt werden, sondern in Einzelfällen eigenständig getroffen werden. Wie es der neue Wortlaut der Mu-RL und auch die Pressemitteilung erläutern, sollen diese Beratung und Aufklärung die Frauen dabei unterstützen, ,eine eigenständige, informierte Entscheidung darüber zu treffen, ob sie diese vorgeburtliche genetische Untersuchung für erforderlich halten“ (G-BA 2019a). Die Beratung hat (gem. MuRL, neue Nr. 3) ,ergebnisoffen“ stattzufinden, mit dem „Ziel einer eigenständigen informierten Entscheidung der Schwangeren“. Die Erläuterung der Bedeutung der Untersuchungsergebnisse hat ,in verständlicher Form“ stattzufinden, wobei auch „die möglichen Folgen einer Entscheidung für diesen Test“ einbezogen werden sollen.

Das bedeutet, dass es (i) keine Checklisten oder Risikoschwellen geben soll, nach denen die Entscheidung getroffen wird, sondern (ii) die Erforderlichkeit der NIPT in der konkreten Einzelsituation (iii) durch die Frauen beurteilt werden soll - (iv) nach umfassender und intensiver, ergebnisoffener Beratung und Aufklärung. Bei dieser Beratung und Aufklärung kann (v) die Frau von ihrem Recht auf vollständiges oder partielles Nichtwissen Gebrauch machen, was im Beratungsgespräch deutlich wer- 
den muss. Im Beratungsgespräch ist zudem (vi) „,der Hinweis zu geben, dass es die Möglichkeit gibt, über die entsprechenden Selbsthilfeorganisationen oder Behindertenverbände mit betroffenen Familien Kontakt aufzunehmen“ (G-BA 2019b, S. 3).

\section{Normative Kontextualisierung}

Zwei Kontexte scheinen uns aus ethischer Sicht besonders wichtig: die Funktion der Mu-RL zur Definition der medizinischen Notwendigkeit nach $\S 135$ Abs. 1 Sozialgesetzbuch (SGB) V und die Voraussetzungen eines nicht rechtswidrigen Schwangerschaftsabbruchs aus medizinischer Indikation nach $\S 218$ a Abs. 2 Strafgesetzbuch (StGB).

Der $\S 135$ Abs. 1 SGB V definiert drei Kriterien, die alle zusammen erfüllt sein müssen, um die Übernahme einer medizinischen Leistung durch die gesetzliche Krankenversicherung (GKV) zu rechtfertigen: Es muss (i) ein diagnostischer und therapeutischer Nutzen nachgewiesen sein; die Maßnahme darf (ii) das medizinisch Notwendige nicht übersteigen; und sie muss (iii) wirtschaftlich sein. Die Liste dieser drei Kriterien ist laut Gesetz vollständig. Insbesondere das zweite Kriterium liegt für die pränatale Diagnostik nicht auf der Hand. Weshalb soll sie medizinisch notwendig sein? Während bei Behandlungen der Sinn des Notwendigkeitskriteriums im Kern der ist, dass nicht mehr bezahlt werden soll, als zur Heilung dieser Krankheit notwendig ist, ist der Zweck, der von der Versorgungsnotwendigkeit vorausgesetzt und in den Raum gestellt wird, im Fall der pränatalen Diagnostik erst zu bestimmen: Welche Zustände sollen als interventionsbedürftig angesehen werden? Der Gesetzgeber hat festgelegt, dass die ,ärztliche Betreuung während der Schwangerschaft“" zum Leistungskatalog der GKV gehört (§ 24d I 1 SGB V) und es ist seit langem anerkannt, dass zu dieser Betreuung auch Vorsorgeuntersuchungen gehören. Der G-BA muss entscheiden, welche Methode dafür die beste ist. In der Regelung des G-BA wird nun für die NIPT festgelegt: Wenn es für eine schwangere Frau notwendig ist, um die Auseinandersetzung mit ihrer Situation im Hinblick auf ihre Schwangerschaft zu ermöglichen, einen NIPT durchzuführen, wird er übernommen. Der interventionsbedürftige Zustand, der die Maßnahme im Sinn der GKVBedingungen rechtfertigt, ist somit das Bedürfnis nach Auseinandersetzung mit ihrer Situation.

Wie Schöne-Seifert et al. (2018) herausgearbeitet haben, besitzt die medizinische Notwendigkeit in der medizinischen Versorgung eine „,zentrale Steuerungsfunktion“ (Schöne-Seifert et al. 2018, S. 334). Das hat zur Folge, dass der G-BA-Beschluss eine doppelte normative Bedeutung hat: Einerseits regelt er die Finanzierung durch die GKV. Andererseits bestimmt er aber (in Gestalt einer medizinischen Richtlinie über die Versorgung während der Schwangerschaft), was zur Grundversorgung gehört und deshalb notwendigerweise Teil guter ärztlicher Praxis ist. - Ein Teil der Skepsis, die in der Öffentlichkeit der Kassenfinanzierung von NIPT entgegengebracht wird, bezieht sich auf die Sorge, dass nicht nur definiert wird, was bezahlt werden muss, sondern auch, was zur guten Praxis gehört. NIPT ist aber ein Instrument, das (u.a.) zum selektiven Schwangerschaftsabbruch benutzt wird. Zur Frage, was zur ,guten Praxis“ gehört, ließe sich vieles sagen. Wir beschränken uns auf einen 
Aspekt, nämlich dass die Geschichte der Pränataldiagnostik und der Ermöglichung eines Schwangerschaftsabbruchs mit medizinischer Indikation in die als ,gut“ angesehene ärztliche Praxis schon viel früher, d.h. seit den 1970er Jahren, begonnen hat und deshalb nicht jetzt erst durch die Integration von NIPT in die Regelversorgung eingeführt wird. ${ }^{2}$ Die G-BA-Regelung muss eine Praxis der Pränataldiagnostik vertretbar gestalten, die aber als solche gesellschaftlich längst etabliert und anerkannt ist.

Zum zweiten Kontext: In den wiedergegebenen Formulierungen der Zugangsvoraussetzungen, vor allem in den „Tragenden Gründen“ (G-BA 2019c), lässt sich unschwer der $\S 218$ a Abs. 2 StGB wiedererkennen, die in Deutschland maßgebliche strafrechtliche Norm über die Zulässigkeit eines Schwangerschaftsabbruchs nach pränataler Diagnostik:

Der mit Einwilligung der Schwangeren von einem Arzt vorgenommene Schwangerschaftsabbruch ist nicht rechtswidrig, wenn der Abbruch der Schwangerschaft unter Berücksichtigung der gegenwärtigen und zukünftigen Lebensverhältnisse der Schwangeren nach ärztlicher Erkenntnis angezeigt ist, um eine Gefahr für das Leben oder die Gefahr einer schwerwiegenden Beeinträchtigung des körperlichen oder seelischen Gesundheitszustandes der Schwangeren abzuwenden, und die Gefahr nicht auf eine andere für sie zumutbare Weise abgewendet werden kann.

Auf diesen letzten Satz muss sich, wie unten weiter ausgeführt, die in den „Tragenden Gründen“ (G-BA 2019c) verwendete Formulierung der ,,zumutbaren Opfergrenze" der Belastung für die Schwangere beziehen. Die Frau muss sich demnach in einer Lage befinden, dass eine mögliche Behinderung ihres Kindes ihren körperlichen oder seelischen Gesundheitszustand schwerwiegend beeinträchtigen könnte. Daraus ergibt sich dann die Dringlichkeit der Klärung durch einen NIPT, ob die entsprechende genotypische Variation vorliegt. Diese normative Rahmung vermeidet konsequent - in Übereinstimmung mit dem heute geltenden $\S 218$ - die embryopathische Indikation oder die Suggestion einer solchen. Nicht die Behinderung oder Krankheit des zukünftigen Kindes darf der Grund für einen Abbruch nach § 218a Abs. 2 sein, sondern die drohende körperliche oder seelische Gesundheitsbeeinträchtigung der Frau. So verstanden, soll der Test also letztlich nicht bloß abklären, ob eine Trisomie vorliegt oder nicht, sondern ob für die Schwangere mit dieser Schwangerschaft (bei der der Fötus eine Trisomie hat oder nicht, worauf man testen kann) eine schwerwiegende Beeinträchtigung ihrer psychischen Gesundheit droht. Wenn diese auch bei Vorliegen einer Trisomie nicht droht, entfällt dieser Grund für die Durchführung (und Finanzierung) des Tests. Ein Grund kann sich aber (s. oben) daraus ergeben, dass die Frau das Nichtwissen über das Vorliegen einer Trisomie für allzu belastend empfindet, auch wenn sie die Schwangerschaft wegen einer Trisomie nicht abbrechen würde. Das Ziel der Klärung, ob diese Schwangerschaft zu einer unzumutbaren Belastung der Frau führen könnte, kann in beiden Fällen nur damit erreicht werden, dass Klarheit über das Vorliegen oder Nichtvorliegen der Trisomie hergestellt wird. Insofern ist der NIPT in einer solchen Situation ,geboten“. NIPT

\footnotetext{
2 Vgl. dazu die Arbeiten von Ilana Löwy (2017 und 2018).
} 
können im Licht des geltenden Schwangerschaftsabbruchsrechts letztlich nicht dazu dienen, wozu sie den Beteiligten zweifellos primär dienen, nämlich zur Feststellung einer chromosomalen Variation und einer möglichen Krankheit oder Behinderung des Kindes, sondern nur dazu, eine schwerwiegende Gefährdung der Gesundheit der Frau abzuklären, die aus dieser chromosomalen Variation entstehen könnte.

Mit dieser Überlegung wird angedeutet, dass die Entscheidung, ob der Test finanziert werden soll, eine Betrachtung der familiären Umstände, der psychischen Verletzlichkeit und der Zumutbarkeiten der schwangeren Frau aus ihrer Sicht voraussetzt. Die Klärung der Zumutbarkeit kann aber im Wesentlichen nur durch die Frau selbst vorgenommen werden. Denn niemand außer sie selbst kann wissen, ob aus der Geburt eines Kindes mit genotypischer Variation oder aus dem Nichtwissen darüber, die Gefahr einer gesundheitlich relevanten Belastung zu entstehen droht.

Es ist dann auch nur konsequent, dass der G-BA darauf verzichtete (wie es einige Teilnehmende des Anhörungsverfahrens gefordert hatten), für die NIPT eine zeitliche Schwelle von 12 Wochen p.c. festzulegen. Wenn NIPT schon von der 9. Woche p.m. an möglich ist (=SSW 7+2), bleiben maximal noch 5-6 Wochen bis zum Ablauf der Frist für einen Abbruch ohne medizinische Gründe. Wenn aber eine unzumutbare Belastung und Gefährdung der seelischen Gesundheit der Frau durch eine genotypische Variation des Kindes droht, so ist dies offensichtlich unabhängig davon, in welcher Woche der Schwangerschaft sich die Frau gerade befindet. Wenn es möglich ist, den NIPT schon früher durchzuführen als die Chorionzottenbiopsie, kann mit NIPT im Fall des Fehlens eines Befundes schon früh klar sein, dass ein invasiver Test nicht notwendig ist. Oder es wird im Fall der Feststellung eines Befundes möglich, den invasiven Test so früh wie möglich anzusetzen. In einer solchen Situation wird der NIPT als Erstlinientest einsetzbar, während er in den anderen Fällen ein Zweitlinientest ist, nach vorangegangenen Screenings. Dass Frauen, die noch innerhalb der 12-Wochenfrist sind, nach einem Befund aus NIPT die Schwangerschaft auch ohne Bestätigung durch eine frühe Chorionzottenbiopsie abbrechen können, ist im geltenden Recht vorgesehen. Wenn einer Frau die Sicherheit ausreicht, die ein NIPT bieten kann, um sich für einen Abbruch innerhalb der Frist zu entscheiden, so liegt dies in ihrem Ermessensspielraum. Wenn man (wie wir es tun) die Fristenregelung unterstützt und davon ausgeht, dass Frauen das Recht haben sollen, nach den für sie relevanten Gründen selbst über die Fortsetzung ihrer Schwangerschaft zu entscheiden, so ist diese Konsequenz nicht zu kritisieren. Es ergibt sich aber eine zusätzliche Verantwortung für das ärztliche Personal, wie auch für die Testanbieter, sehr klar über die Möglichkeit eines falsch positiven Befundes aufzuklären.

Die Spannungen, die in diesem Regelungsmodell bestehen, oszillieren um zwei Achsen: (i) das befürchtete Leiden aufgrund der Geburt eines Kindes mit Trisomie oder des Nichtwissens darüber und (ii) die Bedeutung der Einzelfallentscheidung. Wir erläutern beides. 


\section{Wann ist ein Opfer zumutbar?}

Der G-BA benutzt zur Verdeutlichung seines Arguments im Erklärungstext den Ausdruck ,zumutbare Opfergrenze“:

Die zu befürchtende ,schwerwiegende Beeinträchtigung des seelischen Gesundheitszustandes oder eine Belastung [...] kann angesichts der individuellen Umstände der Schwangeren so schwer und außergewöhnlich sein, dass sie die zumutbare Opfergrenze übersteigt“". (G-BA 2019c, S. 4)

Wie lässt sich bestimmen, ob die Opfergrenze überschritten wird und eine Belastung unzumutbar wird? Vor allem: Wer muss das bestimmen? Um diese Fragen zu klären, muss das Konzept des psychosozialen Leidens genauer betrachtet werden, auf das hier implizit Bezug genommen wird. Einen Hinweis dafür können wir aus der Analyse des Konzeptes eines „,unerträglichen Leidens“ gewinnen, die Claudia Bozzaro und Jan Schildmann (2018) vorgelegt haben. Die Begriffe des Leidens, der Belastung oder ähnlicher Begriffe, wenn sie im Zusammenhang klinischer Richtlinien auftreten, sind demnach in drei Hinsichten erklärungsbedürftig: 1) das Verständnis von Leiden überhaupt und damit verbunden die Frage, wer entscheiden kann, ob ein Leiden unerträglich ist; 2) welche Arten des Leidens bei der fraglichen Entscheidung herangezogen werden können; und 3) die normative Funktion des Leidens. Bei NIPT wird auf eine erwartete oder befürchtete Beeinträchtigung der seelischen Gesundheit oder eine Belastung Bezug genommen, die zu schwer sein kann, um noch als zumutbar zu gelten. Wir können dies ein unzumutbares psychosoziales Leiden nennen, das, so die Argumentation, der Frau aus der Behinderung des Kindes oder aus dem Nichtwissen darüber entstehen könnte.

Für die Klärung der Frage nach der Konzeption von Leiden ist wichtig, ob man von einem subjektiven oder einem objektiven Leidensbegriff ausgehen sollte. Für beide Positionen gibt es Gründe, die Bozzaro (2015) in Auseinandersetzung mit Eric Cassell und Stan van Hooft herausgearbeitet hat. Während bei einer Einschränkung der organischen Funktionsfähigkeit die Annahme noch eher plausibel sein kann, dass Menschen nämlich grundsätzlich das Ziel haben, eine krankheits- oder unfallbedingt verlorene Funktionsfähigkeit wieder zurückzugewinnen (außer sie lehnen eine medizinische Maßnahme bewusst ab), ist die Annahme einer objektiven Beurteilbarkeit der psychosozialen Funktionsfähigkeit im Kontext der Pränataldiagnostik unplausibel. Man kann erstens nicht davon ausgehen, dass Frauen die psychosoziale Belastung aus der Behinderung eines Kindes grundsätzlich für unzumutbar halten. Das kann zweitens auch nicht die Meinung des G-BA sein, weil es sonst keinen Sinn ergeben würde, dass laut G-BA insbesondere vermieden werden soll, dass NIPT in der Form eines Screenings durchgeführt wird. Die Konzeption des psychosozialen Leidens aus einer Behinderung des Kindes muss deshalb eine wesentlich subjektive Konzeption sein, die der betroffenen Frau die Autorität verleiht, das befürchtete Leiden glaubhaft zu machen. Die Frage nach den Typen des Leidens, die eine Rolle spielen können, wird in Übereinstimmung mit dem subjektiven Ansatz von den Mu-RL nicht beantwortet. Es ist die Frau, die eine Antwort darauf finden muss, welche Behinderungen, welche Einschränkungen ihrer Lebenspläne und welche Be- 
lastungen der Familie das befürchtete Leiden bestimmen und welche Aspekte dabei weniger stark ins Gewicht fallen.

Die normative Konsequenz eines als zu schwerwiegend eingeschätzten psychosozialen Leidens ist hier die Übernahme der Kosten für eine NIPT-Untersuchung. Die Problematik dabei ist natürlich die, dass finanziell weniger gut aufgestellte Frauen dadurch in die Situation gebracht werden könnten, auf einen gewünschten NIPT entweder verzichten zu müssen, oder aber, um sich für die Kostenübernahme zu qualifizieren, offen legen müssten, dass die Belastung eine für sie zumutbare Opfergrenze übersteigt.

Diskutieren ließe sich, ob oder inwieweit die Zugangsregel des G-BA ihrer Zielsetzung entspricht, nämlich invasive Tests zu vermeiden. Wenn letztendlich subjektive Kriterien maßgeblich sind, dann ist es sowohl nicht deutlich, ob die Frau im Fall des Fehlens der Möglichkeit von NIPT immer auch ein invasives Testverfahren verlangt hätte, um ihre individuelle Situation zu klären, als auch ob sie nun eine invasive Diagnostik in einer Situation vermeidet, in der sie bisher durchgeführt worden wäre. (Außerdem ist, wie oben bereits gesagt wurde, die Vermeidbarkeit von invasiven Tests durch NIPT dadurch beschränkt, dass die Tests einen unterschiedlichen Testumfang aufweisen.) Die Frau kann dabei ihr eigenes Wohl und Leid mit dem Wohlergehen und Leid des zukünftigen Kindes als eng verbunden ansehen und einen letztendlich zu respektierenden Raum der subjektiven Beurteilung beider Aspekte in Anspruch nehmen, der nicht ganz unabhängig vom Testprozedere gesehen werden kann. Die Mu-RL sehen nur vor, dass sie umfassend informiert wird und davor eine ärztliche Beratung über die medizinischen und die psychosozialen Implikationen der Testentscheidung erhält.

\section{Der Einzelfall und die Einzelfälle}

Es gibt hier einen semantischen Unterschied zwischen dem Singular und dem Plural. Während die Pluralformel ,in Einzelfällen“ eine Einschränkung auf relativ seltene Fälle suggeriert, weist der Singular ,im Einzelfall“ auf eine besondere Qualität der Abklärung hin und macht keine Aussage über die Häufigkeit. In der Pressemitteilung des G-BA heißt es, NIPT können ,in begründeten Einzelfällen“ verwendet werden (G-BA 2019a). Dies deutet auf eine Eindämmungsabsicht hin. Die beschlossene Formulierung der Mu-RL verwendet indes den Singular: „,nach ärztlicher Beurteilung im konkreten Einzelfall“ (G-BA 2019b, Teil B, Nr. 1, Satz 1). Diese Formulierung schließt nicht aus, dass eine Beurteilung bei allen Fällen, die einander in bestimmten Hinsichten ähnlich sind, meistens zum selben Ergebnis kommt.

Der Beschlusstext und nicht die Pressemitteilung ist für die Umsetzung der Regelung maßgeblich; wir orientieren uns deshalb an der Singularformulierung. Was kann es bedeuten, eine Entscheidung ,im konkreten Einzelfall“ zu treffen? Sollen keine allgemeinen Regeln gelten, die für die Beurteilung des Einzelfalls maßgeblich sind? Oder soll der Einzelfall in seiner Singularität schlechthin maßgeblich sein? Es sind jedenfalls mehrere Auslegungen des Prinzips ,,im konkreten Einzelfall“ zu entscheiden, möglich. Allen ist gemeinsam, dass eine Einzelfallentscheidung die besonderen Umstände und Hintergründe einer Situation berücksichtigen soll. Je- 
weils gibt es doch verschiedene Kriterien, die letztendlich unvorhersehbar und nicht als Prinzip vorbestimmt werden können. Dies soll es den Schwangeren möglich machen, in ihrer Situation bestmöglich zu entscheiden. Es kann deshalb mit dem Einzelfall keine bloße Delegation an die Schwangere gemeint sein, zumal es ja auch heißt ,nach ärztlicher Beurteilung im Einzelfall“ (G-BA 2019b, Nr. 1).

In Frage kommen könnte zunächst ein subsumptorisches Modell (vgl. Arras 1994, S. 991 ff.): Dazu müsste der Kanon von Normen und Werten, die im Einzelfall Anwendung finden könnten, bekannt sein. Im Einzelfall wäre nach Maßgabe der Besonderheiten des Falls zu sehen, welche dieser Normen und Werte besonders relevant sind und in welcher Priorität sie angewandt werden sollen. Für die Entscheidung zu NIPT kommen persönliche Maximen oder gruppenspezifische kulturelle Normen in Frage, wie z. B. eine vorher gefestigte Haltung zur Inklusion von Menschen mit Behinderungen oder eine bestimmte Auffassung darüber, welche Gründe für einen Schwangerschaftsabbruch akzeptabel sind. Die Frau versucht dann zu bestimmen, welche ihrer Regeln für den Fall zutrifft.

Eine weitere Auslegungsmöglichkeit der Einzelfallentscheidung wäre, sie in Übereinstimmung mit den normativen Präferenzen der am meisten Betroffenen vorzusehen (Rellensmann 2017). Damit ist eine Entscheidung gemeint, die für die Betroffenen in dem Sinne ,gut“" ist, dass sie von ihnen getragen wird und deshalb vielleicht auch von den anderen respektiert werden kann. Es bleibt in diesem Modell den „Betroffenen“ überlassen, wie weit sie ihre Präferenzen selbst hinterfragen wollen oder können. Die Frau hätte zwar das letzte Wort, wird aber - gemäß ihrer Präferenzen - auch die Personen und die Verhältnisse in ihrer Familie und ihrer Gesellschaft einbeziehen und berücksichtigen, die sie für relevant hält. Das Präferenzenmodell bleibt gegenüber dem Ansatz einer umfassenden Hermeneutik der Situation aber unbefriedigend, weil es die Akteurinnen doch letztlich nur als Präferenzenträgerinnen betrachtet.

Eine an der Kasuistik orientierte Auslegungsmethode betrachtet eine Entscheidungssituation aus allen relevanten Perspektiven und legt großes Gewicht auf die Werte aller Beteiligten (vgl. Arras 1994, S. 1000-1003; Jonsen und Toulmin 1988). Sie nimmt aber an, dass für die entscheidenden Personen immer auch allgemeine Gesichtspunkte wichtig sind, die sich im Konkreten zeigen. Sie müssen in ihrer konkreten Bedeutung geklärt werden. In einem umfassenden Verständnis der Einzelfallentscheidung kann aber nicht davon ausgegangen werden, dass schon bekannt ist, welcher Kanon an Normen und Werten überhaupt herangezogen werden kann. Deshalb schließt diese Vorgehensweise nicht aus, sondern verlangt es geradezu, mit anderen Personen über den Fall zu kommunizieren und sich in der Gesellschaft über Erfahrungen auszutauschen. Die Verständigung darüber, welche allgemeinen Gesichtspunkte (wie z. B. Regeln des Guten und Richtigen) im Fall besonders wichtig sind, welche Fragen gestellt werden müssen, und was sie in Bezug auf NIPT und Behinderungen bedeuten, steht einer Würdigung des Einzelfalls nicht entgegen. Ganz im Gegenteil, sie fördert und ermöglicht sie. Sie beinhaltet es auch, ,für andere" entscheiden zu können, indem die Schwangere von ihren eigenen unmittelbaren Interessen Abstand nimmt und sie in einem reflexiven Prozess mit den Positionen der Anderen vermittelt. 
Das zuletzt beschriebene Modell stellt eindeutig die höchsten Ansprüche. Die Gefahr besteht, dass in den Routinen der vorgeburtlichen Versorgung und auch in der von gesellschaftlichen Erwartungen geprägten Situation der Schwangeren der deliberative Raum nicht zur Verfügung steht, der $\mathrm{zu}$ einer solchen umfassenden Situationsklärung notwendig wäre. Wie empirisch dargelegt wurde, besteht in den westlichen Gesellschaften heute eine hohe Erwartung an die Schwangeren, eine eigene Entscheidung über ihre Schwangerschaft zu treffen. Mianna Meskus (2012) etwa spricht auf Grund einer exemplarischen qualitativen Studie von der Anforderung einer ,personalisierten Ethik“ in Bezug auf die Entscheidungen über das Schicksal des fötalen Lebens, die für die Schwangeren eine auch belastende Aufgabe geworden sei. Es sei, wie Meskus fand, für die Schwangere zunehmend schwierig, sich der Rhetorik der Selbstbestimmung und Autonomie zu entziehen, die das Wissen gegenüber dem Nichtwissen automatisch privilegiert, also das Nichttesten mit dem Makel eines Nicht-Wissen-Wollens belegt. Diese Interpretation wird auch in den vorliegenden Texten des G-BA gestützt, wenn von einer „Auseinandersetzung“ der Schwangeren „mit ihrer individuellen Situation“ die Rede ist (G-BA 2019b, S. 3). Eine Verweigerung der Auseinandersetzung ist gerade im Zweifelsfall schwerer zu rechtfertigen als die Bereitschaft, diese auf sich zu nehmen. Und das bedeutet, den Test im Zweifelsfall eher durchzuführen.

Mit der Betonung des Einzelfallbezugs der Entscheidungen, ob NIPT im Rahmen der Regelversorgung angeboten und durchgeführt werden sollen, soll vermutlich dem Anliegen Rechnung getragen werden, dass NIPT nicht zur blinden Routine werden. ${ }^{3}$ Die Entscheidung soll nicht leichtfertig getroffen werden, sondern in Bezug auf die konkreten Umstände der Situation jedes Mal sorgfältig abgewogen sein, vor allem aus der Perspektive der Schwangeren selbst. Die Betonung des Einzelfalls hat aber gleichzeitig eine legitimatorische Wirkung, weil damit dem sozialethisch formulierten Vorwurf begegnet werden kann, dass NIPT, sobald sie von den Kassen bezahlt werden, „einfach so“ durchgeführt werden, und sich de facto doch ein flächendeckendes Screening auf Trisomie-Schwangerschaften entwickelt.

\section{Ein Paradox oder eine flexible Lösung?}

Wenn man die in der Debatte prominent vorgebrachten Anliegen betrachtet und mit dem Beschluss des G-BA vergleicht, so fällt auf, dass er wegen seiner bewusst paradoxen Struktur keinem dieser Anliegen ganz gerecht zu werden vermag. Wir nennen hier nur die wichtigsten dieser Anliegen:

Die Sorge, dass mit der Finanzierung der NIPT durch die GKV die Wertschätzung behinderter Menschen, insbesondere von Menschen mit Down-Syndrom beeinträchtigt werden könnte (Rüffer 2018; IMEW 2018; BeB et al. 2019), kann durch das Fehlen einschränkender objektiver Kriterien für die Anwendung und Bezahlung von NIPT nicht definitiv beruhigt werden. Die subjektiv empfundene Last der Unwis-

\footnotetext{
${ }^{3}$ Nicht jede Routine muss blind sein; es gibt hilfreiche und ethisch problematische Routinen. Man muss berücksichtigen, dass das extensive Testen in der Schwangerschaftsvorsorge schon längst auf einer ,routine-like expectation“ beruht, an die sich Frauen gewöhnt haben (Meskus 2012, S. 385).
} 
senheit wird schwerer, je größer die Schwierigkeiten sind, die Schwangere für das Leben eines Kindes mit Trisomie befürchten. Je weniger Kinder mit Trisomie geboren werden, desto stärker wird auch der Rechtfertigungsbedarf einer Schwangeren (sich selbst und anderen gegenüber) werden, wenn sie den Test nicht durchführen will.

Der Selbstbestimmung der Frauen wird Raum gegeben, indem sie testen können, wenn es für sie in ihrer Situation ,geboten“ ist. Andererseits ist ein Test aber (wie es die „Tragenden Gründe“ (G-BA 2019c) betonen) vor allem dann erstattungsfähig, wenn es angesichts der Situation um eine für sie selbst gesundheitsrelevante Belastung geht, die die ,zumutbare Opfergrenze“ (G-BA 2019c, S. 4) überschreitet. NIPT als risikoärmere Alternative zu einem bereits kassenpflichtig zugelassenen invasiven Testverfahren wird zwar Kassenleistung, was einer Gerechtigkeitsforderung entspricht (Buyx 2018; Rolfes 2018). Gleichzeitig muss die Schwangere aber jetzt durch die Bezugnahme auf die psychosoziale Indikation nach $\S 218$ a schon früher in der Schwangerschaft - bei der Entscheidung über die Durchführung eines NIPT - über das Auftreten einer für sie unzumutbaren gesundheitsgefährdenden Situation befinden.

Es wurden zwar Regeln formuliert, die den Zugang nach gleichen Kriterien für alle ermöglichen, gleichzeitig wurde aber im wesentlichen Punkt das wiederholt, was aus dem strafrechtlichen Kontext schon klar war, nämlich dass ein Abbruch der Schwangerschaft nach pränataler Diagnose erlaubt ist, wenn eine Behinderung des zukünftigen Kindes die Gesundheit der Frau gefährdet oder belastet. Die Einschätzung, ob dies der Fall ist oder nicht, ist aber mit objektiven Kriterien nicht möglich. Eine solche Regelung kann den Anliegen derjenigen offensichtlich nicht gerecht werden, die für einen weitergehenden Schutz fötalen Lebens eintreten und den selektiven Schwangerschaftsabbruch grundsätzlich kritisieren.

Eine gewisse Paradoxie ist der beschlossenen Reglung nicht abzusprechen. Die Rede von „klar und eindeutig“ geregelten ,sehr engen Voraussetzungen“ (G-BA 2019a) steht einer nur subjektiv bestimmbaren Untragbarkeit der Ungewissheit gegenüber, die das maßgebliche Kriterium ausmacht. Eine nur subjektiv mögliche Bestimmung soll zeigen, ob der Test im Einzelfall „geboten“ ist. Der normative Kontext, in dem sich der Einzelfall in den relevanten Hinsichten erschließt, lässt aber relativ breite Spielräume, die es einer Frau erlauben, den Test dann durchführen zu lassen, wenn er für sie notwendig ist, weil sie am Nichtwissen leidet oder eine für sie unzumutbare Situation befürchtet.

Angesichts der Hitze der vorausgehenden Debatte über die Kassenfinanzierung von NIPT in Deutschland ist es nicht verwunderlich, dass versucht wurde, anfechtbare Positionen weitestgehend zu vermeiden. Der Beschluss deutet aber, genau indem er dies tut, eine Richtung an, in der öffentliche Rechtfertigungen der Durchführung von NIPT (und allgemein der Pränataldiagnostik) in Bezug auf die Situation der Einzelnen gefunden werden können, ohne dabei Kriterien zu formulieren, die, allgemein ausgedrückt, sozialethisch bedenklich wären. Gerade in der Subjektivität seiner entscheidenden Momente könnte der G-BA-Beschluss deshalb für NIPT eine im gesellschaftspolitischen Kontext von Deutschland haltbare pragmatische Lösung darstellen, für die sich auch ethisch argumentieren lässt. 
Damit sind gewiss nicht alle Fragen beantwortet, die sich durch die Regelung des G-BA stellen. Die auf NIPT fokussierte Lösung hat eine möglicherweise wiederum problematische Bevorzugung von NIPT gegenüber anderen nicht finanzierten nichtinvasiven pränataldiagnostischen Verfahren zur Folge, die von den Gesichtspunkten der Vorsorgungsqualität und -gerechtigkeit aus betrachtet werden müssen. Weiterhin ist die emergente soziale Praxis der Pränataldiagnostik nach Einbezug von NIPT in die Versorgungsroutinen von den Gesichtspunkten der Selektion und der Entscheidungsqualität in den Einzelfällen zu bewerten. Ist die routinisierte Anwendung von NIPT, die der G-BA vermeiden will, überhaupt vermeidbar, oder müssten Routinen in der Versorgungspraxis nicht vielmehr so gestaltet werden, dass im Einzelfall gute Entscheidungen getroffen werden können?

Danksagung Diese Arbeit wurde ermöglicht von der DFG (RE 2951/3-1 und SCHU 2846/2-1: Practices of Prenatal Genetic Testing: A Comparative Empirical and Philosophical Study in Germany and Israel, PreGGI). Viele wichtige Hinweise zur Argumentation in diesem Artikel gab uns Hannes Foth; für Anregungen danken wir unseren weiteren Kolleginnen und Kollegen im Projekt Yael Hashiloni-Dolev, Tamar Nov Klaiman, Anika König, Aviad Raz und Stefan Reinsch; für Recherchen im Vorfeld danken wir Zoewend Elodie Kaboré und Johannes Borgmann.

Funding Open Access funding provided by Projekt DEAL.

Open Access Dieser Artikel wird unter der Creative Commons Namensnennung 4.0 International Lizenz veröffentlicht, welche die Nutzung, Vervielfältigung, Bearbeitung, Verbreitung und Wiedergabe in jeglichem Medium und Format erlaubt, sofern Sie den/die ursprünglichen Autor(en) und die Quelle ordnungsgemäß nennen, einen Link zur Creative Commons Lizenz beifügen und angeben, ob Änderungen vorgenommen wurden.

Die in diesem Artikel enthaltenen Bilder und sonstiges Drittmaterial unterliegen ebenfalls der genannten Creative Commons Lizenz, sofern sich aus der Abbildungslegende nichts anderes ergibt. Sofern das betreffende Material nicht unter der genannten Creative Commons Lizenz steht und die betreffende Handlung nicht nach gesetzlichen Vorschriften erlaubt ist, ist für die oben aufgeführten Weiterverwendungen des Materials die Einwilligung des jeweiligen Rechteinhabers einzuholen.

Weitere Details zur Lizenz entnehmen Sie bitte der Lizenzinformation auf http://creativecommons.org/ licenses/by/4.0/deed.de.

\section{Einhaltung ethischer Richtlinien}

Interessenkonflikt C. Rehmann-Sutter und C. Schües geben an, dass kein Interessenkonflikt besteht.

Ethische Standards Für diesen Beitrag wurden von den Autor*innen keine Studien an Menschen oder Tieren durchgeführt. Für die aufgeführten Studien gelten die jeweils dort angegebenen ethischen Richtlinien.

\section{Literatur}

Arras J (1994) Principles and particularity: the role of cases in bioethics. Indiana Law J 69:983-1014

$\mathrm{BeB}$ - Bundesverband evangelischer Behindertenhilfe e. V. et al (2019) Warum wir uns gegen eine Kassenzulassung des nichtinvasiven Pränataltests auf Trisiomie 21 und weitere Trisomien aussprechen! https://beb-ev.de/wp-content/uploads/2019/04/2019-04-10_Argumentationspapier-gegen-NIPT-alsKassenleistung.pdf. Zugegriffen: 7. Juni 2020

Berufsverband niedergelassener Pränatalmediziner e. V., BVNP (2020) Pressemitteilung: NIPT als Kassenleistung: Pränataldiagnostiker sehen gravierende Mängel in G-BA Beschluss. https://www.bvnp. de/media/92-2020-01-22-pm-bvnp-stellungnahme-zu-nipt-januar-2020-f-pdf. Zugegriffen: 7. Juni 2020 
Beta J, Lesmes-Heredia C, Bedetti C, Akolekar R (2018) Risk of miscarriage following amniocentesis and chorionic villus sampling: a systematic review of the literature. Minerva Ginecol 70(2):215-219. https://doi.org/10.23736/S0026-4784

Bornes G (2020) Im Wettbewerb kein Platz für Ethik? Gen-ethischer Informationsd 252:14-15

Bozzaro C (2015) Der Leidensbegriff im medizinischen Kontext: Ein Problemaufriss am Beispiel der tiefen palliativen Sedierung am Lebensende. Ethik Med 27:93-106

Bozzaro C, Schildmann J (2018) "Suffering" in palliative sedation: conceptual analysis and implications for decision making in clinical practice. J Pain Symptom Manage 56(2):288-294

Brauer S, Strub JD, Bleisch B, Bolliger C, Büchler A, Filges I, Miny P, Sax A, Tercanli S, Zimmermann M (2016) Wissen können, dürfen, wollen? Genetische Untersuchungen während der Schwangerschaft. Vdf, Zürich

Braun K, Könninger S (2018) Realizing responsibility. Institutional routines, critical intervention, and the "big" questions in the controversy over non-invasive prenatal testing in Germany. New Genet Soc 37/3:248-267. https://doi.org/10.12688/f1000research.16837.1

Buyx A (2018) Kostenübernahme für pränatale Bluttests. Pro und Contra. Dtsch Arztebl 115:1988

Chiu RWK, Chan KCA, Gao Y et al (2008) Noninvasive prenatal diagnosis of fetal chromosomal aneuploidy by massively parallel genomic sequencing of DNA in maternal plasma. Proc Natl Acad Sci USA 105:20458-20463

Dohr A, Bramkamp V (2014) Nicht invasive Pränataltests NIPT. In: pro familia (Hrsg) pro familia medizin. der familienplanungsrundbrief. Nr. 2 (Mai 2014). https://www.profamilia.de/fileadmin/ dateien/fachpersonal/familienplanungsrundbrief/pro_familia_medizin_2_2014.pdf. Zugegriffen: 4. Febr. 2019

Fan HC, Blumenfeld YJ, Chitkara U et al (2008) Noninvasive diagnosis of fetal aneuploidy by shotgun sequencing DNA from maternal blood. Proc Natl Acad Sci USA 105:16266-16271

Gemeinsamer Bundesausschuss (2016a) Richtlinien des Gemeinsamen Bundesausschusses über die ärztliche Betreuung während der Schwangerschaft und nach der Entbindung (,Mutterschafts-Richtlinien“). https://www.g-ba.de/downloads/62-492-1223/Mu-RL_2016-04-21_iK-2016-07-20.pdf. Zugegriffen: 11. Jan. 2019

Gemeinsamer Bundesausschuss (2016b) Antrag auf Bewertung der Methode der nicht-invasiven Pränataldiagnostik (NIPD). https://www.g-ba.de/downloads/40-268-3933/2016-08-18_EinleitungBeratungsverf_nicht-invasive-Praenataldiagnostik_Antrag.pdf. Zugegriffen: 10. Jan. 2019

Gemeinsamer Bundesausschuss (2019a) Pressemitteilung Nr. 26/2019. Nicht-invasiver Test zum Vorliegen von Trisiomien als mögliche Alternative zu invasivem Eingriff. https://www.g-ba.de/downloads/34215-810/26_2019-09-19_Mu-RL_NIPT.pdf. Zugegriffen: 7. Juni 2020

Gemeinsamer Bundesausschuss (2019b) Beschluss des Gemeinsamen Bundesausschusses über eine Änderung der Mutterschafts-Richtlinien (Mu-RL). https://www.g-ba.de/downloads/39-261-3955/201909-19_Mu-RL_NIPT_BAnz_WZ.pdf. Zugegriffen: 7. Juni 2020 (Nicht-invasive Pränataldiagnostik zur Bestimmung des Risikos autosomaler Trisomien 13, 18 und 21 mittels eines molekulargenetischen Tests (NIPT) für die Anwendung bei Schwangerschaften mit besonderen Risiken vom 19. September 2019. BAnz AT 20.12.2019 B6)

Gemeinsamer Bundesausschuss (2019c) Tragende Gründe zum Beschluss des Gemeinsamen Bundesausschusses über eine Änderung der Mutterschafts-Richtlinien [...] vom 19. September 2019. https:// www.g-ba.de/downloads/40-268-6007/2019-09-19_Mu-RL_NIPT_TrG.pdf. Zugegriffen: 7. Juni 2020

Hill C, Compton C, Karunaratna M, Lewis C, Chitty L (2014) Client views and attitudes to non-invasive prenatal diagnosis for Sickle Cell Disease, Thalassaemia and Cystic Fibrosis. J Genet Couns 23:1012-1021

Hoyer J (2018) Indikationen und Inhalte der humangenetischen Beratung. Frauenheilkd Up2dat 12(1):55-71

Huster S (2017) Der Gemeinsame Bundesausschuss als Ethikbehörde? Medizinrecht 35:282-286

Institut für Qualität und Wirtschaftlichkeit im Gesundheitswesen (IQWiG) (2018) Nicht invasive Pränataldiagnostik (NIPD) zur Bestimmung des Risikos autosomaler Trisomien 13, 18 und 21 bei Risikoschwangerschaften - Abschlussbericht vom 30.04.2018. https://www.iqwig.de/ de/projekte-ergebnisse/projekte-301/nichtmedikamentoese-verfahren/s-projekte/s16-06-nichtinvasive-praenataldiagnostik-zur-bestimmung-des-risikos-autosomaler-trisomien-13-18-und-21bei-risikoschwangerschaften.7776. Zugegriffen: 7. Juni 2020

Institut Mensch-Ethik-Wissenschaft IMEW (2018) Partizipation in technisch-gesellschaftlichen Innovationsprozessen mit fragmentierter Verantwortung am Beispiel der nicht-invasiven Pränataldiagnostik. Selbstverlag IMEW, Berlin (https://www.imew.de/fileadmin/Dokumente/Volltexte/Tagungen_2018/ IMEW-Projekt-NIPD-Kurzfassung_2018.pdf. Zugegriffen: 4. Feb. 2019) 
Jonsen AR, Toulmin S (1988) The abuse of casuistry. A history of moral reasoning. University of California Press, Berkeley

Lo YMD, Corbetta N, Chamberlain PF (1997) Presence of fetal DNA in maternal plasma and serum. Lancet 350:485-487

Löwy I (2017) Imperfect pregnancies. A history of birth defects and prenatal diagnosis. Johns Hopkins University Press, Baltimore

Löwy I (2018) Tangled diagnosis. Prenatal testing, women, and risk. University of Chicago Press, Chicago

Meskus M (2012) Personalized ethics: the emergence and the effects in prenatal testing. BioSocieties 7(4):373-392

Pös O, Budiš J, Szemes T (2019) Recent trends in prenatal genetic screening and testing. F1000Research 8(F1000 Faculty Rev):764. https://doi.org/10.12688/f1000research.16837.1

Ravitsky V (2017) The shifting landscape of prenatal testing: between reproductive autonomy and public health. Hastings Cent Rep 47(Suppl 3):S34-S40

Rellensmann G (2017) Ethische Herausforderungen in der Neonatologie und Intensivmedizin. Pädiatrie 29(51):26-30

Richter-Kuhlmann E (2019) Einzelgenerkrankungen: Neuer pränataler Bluttest kommt auf den Markt. Dtsch Arztebl 116(42):A-1870 (B-1542 / C-1510)

Rolfes V (2018) Aspekte der Gerechtigkeit in der Pränataldiagnostik am Beispiel der nicht-invasiven Tests. In: Steger F et al (Hrsg) Pränatalmedizin. Alber, München, S 52-67

Rüffer C (2018) Kostenübernahme für pränatale Bluttests. Pro und Contra. Dtsch Arztebl 115:1989

Sacco A, Hewitt H, Pandya P (2019) Women's choices in non-invasive prenatal testing for aneuploidy screening: results from a single centre prior to introduction in England. Arch Dis Child. https://doi. org/10.1136/archdischild-2019-317031

Schmitz D (2013) A new era in prenatal testing: are we prepared? Med Health Care Philos 16:357-364

Schöne-Seifert B, Friedrich DR, Harney A, Huster S, Raspe H (2018) „Medizinische Notwendigkeit“: Herausforderungen eines unscharfen Begriffs. Ethik Med 30:325-341

Steger F, Orzechowski M, Schochow M (Hrsg) (2018) Pränatalmedizin. Ethische, juristische und gesellschaftliche Aspekte. Alber, München 